\title{
Práticas acadêmicas, cirurgióes-dentistas, população e câncer bucal
}

\author{
A análise e a compreensão das práticas e atitudes dos \\ cirurgiões-dentistas e da população são importantes para que se \\ consiga diagnosticar o câncer bucal precocemente.
}

\author{
Izabella Barison Matos*, Lenita Aver Araújo** \\ * Docente da UNIPLAC/Lages(SC). \\ ** Docente da UFPel/Pelotas (RS).
}

\section{RESUMO}

A investigação sobre conhecimentos, práticas e atitudes em relação ao diagnóstico do câncer bucal na visão da população e do cirurgião-dentista, realizada em 2002, em Lages (SC), foi empreendida com a intenção de aproximar os alunos do curso de Odontologia, da UNIPLAC, da realidade social e cultural da população e sensibilizá-los a valerem-se de conhecimentos de outras áreas para entender o atraso do diagnóstico de câncer bucal. Análises dos dados obtidos podem explicar por que a doença continua sendo diagnosticada nas fases mais avançadas. De um lado, os cirugiões-dentistas não realizam um correto exame clínico, que é um método eficaz para seu diagnóstico; do outro, a população não percebe as lesões precursoras, pois são assintomáticas e indolores. É bom lembrar o perfil da população vulnerável à doença: homens entre 50-70 anos, fraco capital escolar e econômico, que não têm o hábito de “cuidar de si”. É preciso entender que, para este segmento populacional, são mais facilmente identificadas, aquelas manifestações mais visíveis e concretas, que caracterizam estágios avançados da doença. Assim, programas e ações direcionadas ao câncer bucal deverão considerar atitudes, valores, crenças da "população-alvo" para que a abordagem preventiva e educativa seja mais eficaz.

\section{DESCRITORES}

Câncer bucal. Sociologia médica. Diagnóstico. Prevenção primária.
A s concepções e representações sociais do patológico, dos estados mórbidos, da doença e da cura não se reduzem ao discurso dos profissionais da área da Saúde (BOLTANSKI, 1984; DORVIL, 1985; HERZLICH, 1969; LOYOLA et al., 1984; MARSíGLIA, 1998). Assiste-se, nos últimos anos, ao surgimento de um novo enfoque: o discurso sobre a saúde e a doença sob o ponto de vista dos usuários, do doente. A partir dessa perspectiva, a percepção dos processos mórbidos da doença remete às subjetividades individuais; sugere-se, portanto, que as doenças e suas representações sociais estejam intimamente ligadas e dependentes do tipo de sociedade em que são produzidas. Reflexões mais abrangentes, ligadas às representações populares sobre a etiologia, sintomatologia, tratamento e prevenção do câncer, têm permitido conhecer algumas práticas de higiene oral empregadas pela população (MIYACHI et al., 2002; UNFER, SALIBA, 2000).

O perfil do portador de câncer bucal é esboçado da seguinte forma: homens, com idade entre 45 e 55 anos, etilistas e tabagistas (LEITE, 1997). Uma das possíveis explicações para o fenômeno, ou seja, o fato de se ter mais homens que mulheres com câncer bucal em estágios avançados da doença, pode ser a de que, segundo a literatura sociológica, as mulheres demonstram ser mais atentas que os homens às suas sensações e percepções. Dizendo de outra forma, elas "se observam" mais que os homens, pois mantêm uma relação de atenção e cuidados com seu corpo (BOLTANSKI, 1984; BORDIEU, 1977; DORVIL, 1985; LOYOLA et al., 1984). 
O interesse deste estudo centra-se na identificação, análise e compreensão das práticas e atitudes dos cirurgiões-dentistas e da população acerca do câncer bucal. Em relação aos primeiros pretendeu-se saber sobre seu preparo e suas práticas profissionais diante do câncer bucal. Já com a população, o interesse foi identificar a percepção do câncer bucal e a formação do itinerário terapêutico relativo à doença. Com os alunos do curso de Odontologia da UNIPLAC, o objetivo foi aproximá-los da realidade social e cultural da população e sensibilizá-los a valerem-se de conhecimentos das ciências sociais para entender o atraso do diagnóstico de câncer bucal. Análises dos dados obtidos podem explicar por que a doença continua sendo diagnosticada nas fases mais avançadas.

\section{MATERIAL E MÉTODOS}

O estudo foi realizado entre abril e dezembro de 2002, na cidade de Lages, município situado no Planalto Sul do Estado de Santa Catarina. Segundo o IBGE (2001), a população da cidade é de 157.682 habitantes, 76.620 homens $(48,59 \%)$ e 81.062 mulheres $(51,41 \%)$, majoritariamente, moradores no espaço urbano. A população está distribuída em $5.287 \mathrm{~km}^{2}$ e $28 \%$ da mesma encontra-se na faixa etária entre 40-60 anos de idade.

Como instrumento de pesquisa foram utilizados questionários com perguntas fechadas, abertas e semi-abertas, sendo um direcionado aos cirurgiões-dentistas e outro à população. A estrutura dos questionários, semelhante à da pesquisa realizada na Universidade Federal de Pelotas (RS), foi adaptada aos objetivos e particularidades do presente estudo.

Decidiu-se pela definição de uma amostra de $48 \%$ desses profissionais, totalizando 70 cirurgiões-dentistas, registrados na Associação Brasileira de Odontologia/ABO - seccional da Serra Catarinense. Esse número não incluiu profissionais de duas categorias (cirurgiões-dentistas docentes da UNIPLAC e odontopediatras docentes e não-docentes da UNIPLAC), que não interessavam às pesquisadoras.

Responderam ao questionário 675 pessoas (adultos), residentes em 19 (em média, foram aplicados 35 questionários por bairro) dos 69 bairros de Lages, cuja aplicação foi feita por alunos do curso de Odontologia da UNIPLAC, conforme previsto.

Em dois momentos, antes da aplicação dos questionários, os alunos foram orientados sobre a pesquisa e a atuação na execução do projeto. Uma alu- na-bolsista coordenou o recebimento dos envelopes com os questionários respondidos. Houve a pré-testagem e alguns ajustes foram feitos, bem como explicações aos alunos-aplicadores.

\section{RESULTADOS \\ Perfil dos cirurgiões-dentistas pesquisados}

Perfil: gênero masculino, 40 anos, colou grau em universidade federal, tem aperfeiçoamento (prioritariamente em Dentística, Periodontia ou Ortodontia) e há cerca de vinte anos no mercado, atuando em consultório particular.

Entre os cirurgiões-dentistas, $77 \%$ consideram-se preparados para diagnosticar lesões cancerígenas. No entanto, somente $44 \%$ costumam fazê-lo. Quase a totalidade dos cirurgiões-dentistas $(97 \%)$ informaram que estão em processo constante de atualização, sendo que a leitura de artigos científicos recebeu o maior percentual de indicação $(77,9 \%)$, seguido de livros $(76,7 \%)$, participação em congressos $(54,4 \%)$, palestras $(51,5 \%)$ e cursos $(48,5 \%)$.

Respostas à pergunta, referente à apresentação clínica de lesões bucais, que podem ser câncer, demonstram que somente $27,2 \%$ apontaram a opção "úlcera" (que é a apresentação clínica do câncer bucal, mais freqüente). A opção vesícula/bolha teve $6,7 \%$ de indicações.

\section{O perfil da população e o itinerário terapêutico}

Das 675 pessoas entrevistadas, $58,8 \%$ pertencem ao gênero feminino e 41,2\% são homens. Grande parte das mulheres não exercia atividade remunerada fora de casa. No contingente feminino, 11,6\% são "domésticas" e 3,2\% "costureiras", entre outras ocupações. Entre os homens, encontraram-se ocupações características do segmento pesquisado: aposentado, estudante, pedreiro, comerciante, servente, eletricista, motorista, vendedor, para explicitar os mais citados.

A maioria das pessoas pesquisadas detém fraco capital escolar: 9,3\% não freqüentaram a escola, mas sabe ler e escrever; 2,7\% são analfabetos; $65 \%$ não concluíram o ensino fundamental e somente $23 \%$ têm diploma desse mesmo nível de ensino. Quanto à faixa etária dos entrevistados: 32,9\% têm menos de 30 anos; 25,9\% têm entre 30 e 39 anos de idade; $18,8 \%$ estão na faixa etária de 40-49 anos e 22,4\% têm mais de 50 anos.

Pode-se dizer que, em relação ao capital econômico, $52 \%$ das famílias têm uma renda mensal bai- 
$x a$, de $R \$ 200,00$ a $R \$ 600,00$ (na época da pesquisa). Para $48 \%$ das famílias dos respondentes, há 3 ou 4 dependentes e $29 \%$ têm de 5 a 6 membros que dependem da renda familiar.

Ao se solicitar que citassem doenças que podem ocorrer na boca, 39,4\% disseram "não saber"; 15,4\% apontaram o "câncer" e 15,3\% afta, para exemplificar as indicações com maior freqüência. Entre aqueles que disseram ter tido alguma das doenças citadas, ao se indagar qual atitude tomaram, responderam: "procurou profissional" (25,3\%); apelou para "medicação caseira" $(18,7 \%)$ ou para "medicação de farmácia” $(18,7 \%)$ e $25,5 \%$ indicaram "outros".

A pergunta referente a que profissional foi procurado apresentou, segundo a freqüência: "nenhum" (51,3\%), "dentista" (23,5\%), "farmacêutico" $(15,2 \%)$ e "médico" (10\%). Ao passo que, a questão "Se tivesse alguma ferida/doença/caroço na boca, quem procuraria?" mostrou que entre os 675 pesquisados, $41,2 \%$ indicaram o médico, $37,3 \%$ o dentista e o "farmacêutico" (atendente de farmácia) teve 7\% das indicações.

Entre os respondentes, 70,2\% acham que o dentista conhece e sabe tratar das doenças da boca, porque "trata dos dentes", "estudou para isso" ou simplesmente "porque sim". Já, 26,4\% entendem que "não", pois "não é médico" ou "só trata dos dentes". Em relação à etiologia do câncer, apesar da desinformação constatada por perguntas anteriores, citaram o fumo e o álcool como causadores do câncer. Ou seja, 37,6\% não sabem, 24,7\% apontaram o cigarro, $8,4 \%$ citaram a combinação cigarro e álcool, para apresentar os mais citados.

Sobre a sintomatologia ou "Que tipo de sinal ou sintoma mostra que a pessoa tem câncer de boca"?, $50,5 \%$ não sabem identificar os sintomas; $13,8 \%$ citam "dor" e 11,4\% "feridas". É importante ressaltar que, dor e ferida fazem parte do quadro de estágios mais avançados da doença e não de aspectos clínicos característicos do estágio inicial, que são indolores e assintomáticos.

\section{DIscussão}

\section{O cirurgião-dentista e o câncer bucal}

A grande maioria dos cirurgiões-dentistas pesquisadores considera-se preparada para diagnosticar lesões cancerígenas. No entanto, somente a metade costuma fazê-lo. Quase a totalidade dos cirurgiões-dentistas informou que está em processo constante de atualização, sendo que a leitura de artigos científicos recebeu o maior percentual de indicação, seguido pelos livros, participação em congres- sos, palestras e cursos.

Com tal nível de informação poderia-se supor que os dentistas deveriam estar preparados, teoricamente, para o diagnóstico de doenças bucais. Mas, as respostas à pergunta referente à apresentação clínica de lesões bucais que podem ser câncer demonstram que muito poucos apontaram a opção "úlcera" (que é a apresentação clínica do câncer bucal, mais freqüente). E o mais preocupante: a opção vesícula/bolha, que teve algumas indicações, não deveria ter sido apontada, pela total impossibilidade de existir uma relação entre ela e o câncer.

Pode-se dizer que apesar de os cirurgiões-dentistas considerarem-se aptos a fazer diagnósticos de doenças bucais, as respostas às perguntas do questionário revelam pouca familiaridade e uma certa dose de despreparo, já detectadas em outras pesquisas. Percebe-se grande distância entre o dizer desses profissionais e a sua prática coditiana.

Parece que algumas análises sobre o modelo de ensino da Odontologia podem ser elucidativas para entendermos o que ocorre. Comumente acredita-se que basta capacitar para que a ação pedagógica se reflita num bom desempenho. No entanto, argumenta-se que a mera implementação de um currículo de Odontologia sintonizado com as necessidades sociais e de saúde bucal da população não resolverá por si só a questão (SANTANA, CHRISTÓFARO, 2002; SANTANA et al., 2002).

\section{A população e o câncer bucal: alguns comentários}

O material coletado para análise permitiu observar que quanto maior o capital escolar dos respondentes, maior sua tendência em buscar o dentista, como o profissional certo para tratar de alguma patologia bucal. Por outro lado, o reduzido capital escolar dos pesquisados evidencia uma certa limitação no discurso, uma vez que é a escola que oferece os esquemas explicativos que favorecem a compreensão e sistematização da linguagem. Associadas a essa falta de aptidão, pode-se identificar uma consciência aguda da ignorância, que os faz hesitar em descrever suas sensações (BOLTANSKI, 1984).

Uma das possíveis explicações para o fenômeno, ou seja, o fato de se ter mais homens que mulheres com câncer bucal em estágios avançados da doença, pode ser a de que, segundo a literatura sociológica, as mulheres demonstram ser mais atentas do que os homens às suas sensações (de dor ou bem-estar, por exemplo) e percepções. Dizendo de outra forma, elas "se escutam" mais que os homens, pois "mantém 
uma relação de atenção e cuidados com seu corpo" (BOLTANSKI, 1984; DORVIL, 1985).

Por meio de, principalmente Dorvil, há indicações de estudos realizados por cientistas sociais, na década de 80 , em que as classes populares não têm uma relação reflexiva com o corpo, pois essa relação de reflexividade é própria das

\footnotetext{
“"classes superiores' e é pouco compatível com uma utilização intensa do corpo (...). A percepção da dor e mais freqüentemente das sensações corporais é igualmente aguçada em diferentes classes sociais. (...) Elas são objeto de uma seleção e são vivenciadas diferentemente com maior ou menor intensidade segundo a classe social a que pertencem".
}

Em relação às práticas preventivas (SANTANA, CHRISTÓFARO, 2002; VERDÈs-LEROUX, 1986), não se pode crer na possibilidade de uma correspondência entre o nível de informações sobre um determinado problema e o comportamento da pessoa perante ele. Isto porque, o enfoque comportamental é insuficiente para atender as expectativas dos profissionais de Saúde e levar os diversos "públicos-alvo" a mudar suas práticas cotidianas. Identificam-se dois componentes que dificultam a comunicação: autoritarismo e pressa. As atitudes autoritárias de grande parte dos trabalhadores da Saúde, diante de pessoas das classes populares, são uma característica do exercício dos profissionais de Saúde. Estudos têm mostrado que os discursos direcionados à prevenção são assistidos de forma passiva pela população (VERDÈS-LEROUX, 1986).

Parece acertado dizer que, na maioria das vezes, as intervenções são tentativas de inculcação de comportamentos julgados necessários e de forçar uma determinada adesão. Assim, para melhorar o nível de compreensão e as práticas preventivas sobre as doenças da boca, é preciso identificar os fatores que motivam cada pessoa e que condicionam suas atitudes e práticas terapêuticas.

Pesquisas têm mostrado que raramente o câncer bucal é identificado no seu estágio inicial; cerca de 60 a $80 \%$ das lesões são diagnosticadas nos estágios avançados o que reduz a chance de sobrevida de 80\% para 18\% (PIRES et al., 2000; MIYACHI et al., 2002; DAVID et al., 2000).

Num estudo realizado em Curitiba, os pesquisadores informam que o câncer de boca é o $6^{\circ}$ mais freqüente nos homens e o $8^{\circ}$ nas mulheres e $60 \%$ dos casos são diagnosticados em estágios avançados. Em 2001, esse tipo de câncer foi responsável por 50 óbi- tos (MrYACHI et al., 2002). O perfil do paciente com câncer bucal é, geralmente, constituído por 86,07\% de homens, com idade entre 45 e 55 anos e tabagistas $(95,08 \%)$ (MOREIRA, KIGNEL, 1998).

Da mesma forma, em trabalho de extensa revisão da literatura acerca de fatores de risco para tumores de boca e faringe e análise da incidência e mortalidade por essas neoplasias no Brasil, baseada nos Registros de Câncer de Base Populacional, LEITE ${ }^{10}$ retoma um discurso recorrente entre os estudiosos do câncer bucal, que insistem em dizer que o "diagnóstico é simples". Nesta direção, afirma outra pesquisadora que o exame clínico é um dos procedimentos mais simples (JITOMIRSKI, 2000).

Segundo o site www.oralcancer.npg.ig.com.br. "O auto-exame é um método simples”. Da mesma forma, no site do Hospital do Câncer, lê-se:

\footnotetext{
"Apesar da facilidade com que o diagnóstico de muitos tumores - como os de pele e boca - poderia ser feito, a maioria deles é diagnosticado quando o tumor apresenta dimensões superiores a $2 \mathrm{~cm}$ ".
}

TORRES, num trabalho de levantamento bibliográfico sobre a relação histórica da Odontologia e o câncer bucal no País, discute o problema e traça o perfil do cirurgião-dentista para o enfrentamento dessa neoplasia. O autor vai dizer que a preocupação em relação ao câncer de boca remonta à década de 30 "quando Mário Kroeff, proferindo palestras, buscava conscientizar os cirurgiões-dentistas de seu importante papel na detecção de lesões iniciais dessa patologia" (TORRES, 1992). No seu artigo, descreve as iniciativas, a partir daquele marco inicial. Porém, sabe-se que a técnica de exame bucal comprobatória da detecção de "anormalidades" na boca "não requer conhecimentos de alta complexidade, típicos dos profissionais de nível superior. A complexidade não está em reconhecer uma anormalidade, mas sim em obter o diagnóstico confirmatório da lesão cancerígena" ${ }^{\text {. }}$.

Assim, reafirma-se que, a prevenção e o diagnóstico precoce podem ser realizados pelo cirurgião-dentista por meio de um correto exame clínico. PARAJARA, 1999, em pesquisa realizada, afirma que os cirurgiões-dentistas "não se julgam responsáveis pela resolução do problema”. Bem como, não sabem orientar e muito menos estimular o paciente para prevenir-se.

Mas, conforme já assinalado, o estudante de Odontologia, na sua formação acadêmica, recebe ensinamentos de Patologia Bucal e de Estomatolo- 
gia, que o habilitam ao diagnóstico e tratamento das doenças que acometem a boca. Em nenhuma especialidade médica há, no conteúdo programático curricular, tal embasamento, o que faz com que o dentista seja o profissional capacitado para tanto.

\section{CONCLUSÕES}

Com a colaboração da sociologia da saúde observou-se que a percepção dos processos mórbidos remete às subjetividades individuais; essa abordagem considera o conjunto de valores, normas, regras sociais e modelos culturais dos pesquisados.

Tal ponderação permite compreender, em parte, que, pela sua condição social, esses representantes de um segmento das classes populares, sejam inábeis em transmitir informações do seu estado mórbido. Mesmo porque, conforme já foi dito, não prestam muita atenção ao seu corpo, pois percebem-no como instrumento de trabalho.

Uma das conseqüências de não prestarem muita atenção em si, no seu corpo, faz com que os homens só identifiquem as manifestações de neoplasias, quando as dores e o mal-estar lhe impossibilitem o uso instrumental do corpo. Essa atitude pouco reflexiva, característica desse segmento populacional, pode ser uma das explicações possíveis para a compreensão do perfil dos portadores de câncer bucal.

Considerando-se que os pesquisados detêm fraco capital escolar, compreende-se que somente o que pode ser percebido, o concreto, constitua-se num sinal da doença. Conforme já explicitado, faltam-lhes instrumentos de apropriação simbólica, veiculados pela escola, para melhor definir ou perceber sinais não tão evidentes, como aqueles menos identificados por eles. As alterações, dentro da lógica das classes populares, devem ter visibilidade e concretude.

Apesar do diagnóstico ser considerado "muito fácil", pelos estudiosos do assunto, sabe-se que a doença continua sendo diagnosticada nas fases mais avançadas. Da mesma forma, diz-se que o auto-exame é um "método simples", mas a população não tem essa prática incorporada. Querer que a população realize auto-exame de boca, a fim de possibilitar que lesões precursoras do câncer (mudança de cor da pele, úlcera, inchações, por exemplo) sejam identificadas como sinais e sintomas da doença, não é uma tarefa nada simples ou fácil. Isto porque, a população percebe muito mais facilmente aquelas manifestações mais visíveis e concretas, que caracterizam estágios avançados da doença: dificuldade de mastigar, de falar, emagrecimento rápido, dores, por exemplo.
Por um lado, a ignorância sobre a doença, de boa parcela da população, é apontada como um dos fatores para o atraso do diagnóstico, como também, não se pode esquecer das especificidades desse segmento, que não percebe as lesões precursoras, que são assintomáticas e indolores. Por outro, os cirurgiões-dentistas não realizam um correto exame clínico, que é o método eficaz para seu diagnóstico.

Ressalte-se aqui, que não são necessários conhecimentos profundos ou emprego de alta tecnologia para o cirurgião-dentista detectar a patologia. Bastam os conhecimentos básicos obtidos durante o curso de graduação. Assim, uma outra análise a ser retomada refere-se às discussões sobre a relação entre a universidade, como formadora de recursos humanos, o mercado de trabalho e as necessidades epidemiológicas da população. Para alguns, cabe à universidade proporcionar as credenciais necessárias para o bom desempenho profissional. Para outros, a simples implementação de currículos sintonizados com as necessidades da população não vai resolver as contradições e desencontros existentes, explicitados brevemente neste trabalho.

Portanto, os programas a serem criados, visando ações curativas, preventivas e educativas, bem como o planejamento em Odontologia, direcionados às doenças bucais deverão considerar as atitudes, valores, crenças, enfim, as representações sociais acerca das doenças bucais. A partir daí será possível iniciar um trabalho para modificar o atual quadro, pois a abordagem preventiva e educativa deve ser mais eficaz em relação ao câncer bucal. Isso porque, também, evidenciou-se que, para muitos da população pesquisada, o câncer bucal não é percebido como uma doença cujo tratamento e cura compete aos cirurgiões-dentistas.

\section{ABSTRACT}

Academic practices, the dentist, the population and oral cancer

This inquiry into knowledge, practices and attitudes in relation to the diagnosis of oral cancer from the perspectives of the population and of the dentist, carried out in 2002, in Lages county (SC), was undertaken with the intention of creating awareness in the students of the course of dentistry, University of the Planalto Catarinense, about the social and cultural reality of the population, and to sensitize them so that they will use knowledge from other areas to understand the delay in the diagnosis of oral cancer. Analyses of the collected data can explain why this illness continues being diagnosed in the most advan- 
ced phases. On one hand, dentists do not carry out correct clinical examinations, which is the most efficient method for its diagnosis; on the other, the population does not perceive the precursory lesions, therefore they are without symptoms and pain. It is good to remember the profile of the vulnerable population to the illness: men between 50-70 years, with little formal education and low socioeconomic status, that do not have the habit of "taking care of themselves". It is necessary to understand that, for this population segment, those more visible and concrete manifestations, which characterize advanced stages of the illness, are more easily identified. Thus programs and actions directed at oral cancer will have to consider attitudes, values and beliefs of the "target population" so that preventive and educative evaluations can be more efficient.

\section{DESCRIPTORS}

Oral cancer. Sociology, medical. Diagnosis. Primary prevention.

\section{REFERÊNCIAS BIBLIOGRÁFICAS}

AUGÉ, M.; HERZLICH, C. Le ens du mal: anthropologie, histoire, sociologie de la maladie. Paris : Editions des Archives Contemporaines, 1986. p. 217-256.

BOLTANSKI, L. As classes sociais e o corpo. Rio de Janeiro : Graal, 1984.

BOURDIEU, P. Remarques provisoires sur la perception du corps. In: Actes de la Recherche en Sciences Sociales. Paris : Minuit. n. 1, 1977. p. 51-54.

CENTRO DE TRATAMENTO E PESQUISA - HOSPITAL DO CÂNCER - A. C. CAMARGO. Disponível em: http:/www.hcanc.org.br. Acesso em: 11/12/2002.

DAVID, F. P. et al. Avaliação dos conhecimentos, atitudes e práticas do CD diante do câncer bucal. Trabalho apresentado no $5^{\circ}$ OdontoRio 2000. Disponível em http://www.odontolo-

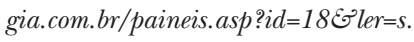

DORVIL, H. Types de societés et de répresentation du normal et du pathologique: la maladie physique, la maladie mentale. In: DUFRESNE, J.; DUMOND, F.; MARTIN, Y. (orgs.). Traité d'Anthropologie Medicale: I'instituition de la santé et de la maladie. Canadá/France: Presses de L'Université du Quebéc/Presses Universitaires de Lyon, 1985. p. 305-332.

GARRAFA, V.; MOYSÉS, S. J. Odontologia brasileira: tecnicamente elogiável, cientificamente discutível, socialmente caótica. Divulgação, n. 13, p. 6-17, jul. 1996.

HERZLICH, C. Santé et maladie. Analyse d'une Representation Sociale. Paris : Mouton, 1969.

JITOMIRSKI, F. Câncer bucal. In: PINTO, V. G.. Saúde bucal coletiva. São Paulo : Livraria Santos Editora, 2000. p. 445-456.

LEITE, I. C. G. Câncer de boca e faringe: estudo dos fatores de risco e análise de sobrevida de uma coorte hospitalar. São Paulo, 1997. Dissertação (Mestrado) - Fiocruz/ENSP.
LOYOLA, M. A. et al. Médicos e curandeiros: conflito social e saúde. São Paulo : Difel, 1984.

MARSÍGLIA, R. M. G. Perspectivas para o ensino das Ciências Sociais na graduação odontológica. In: BOTAZZO, C.; FREITAS, S. F. T. (orgs.) Ciências Sociais e saúde bucal. São Paulo : Editora UNESP, 1998. p. 175-196.

MINAYO, M. C. O conceito de representações sociais dentro da sociologia. In: GUARESCHI, P.; JOVCHELOVITCH, S. (orgs.) Textos em representações sociais. Petrópolis : Vozes, 1995. p. 89-111.

MINISTÉRIO DA SAÚDE. INCA. Estimativa da incidência e mortalidade por câncer no Brasil. 2002. Disponível em: http://www.inca.gov.br/. Acesso em: 11/12/2002.

MIYACHI, S. et al. Centro de Diagnóstico de Lesões Bucais: potencial impacto na epidemiologia do câncer de boca em Curitiba. Rev Bras Cir Implant, v. 9, n. 33, p. 80-85, jan./mar. 2002.

MOREIRA, C. A.; KIGNEL, S. Prevenção do câncer bucal. Rev Assoc Paul Cir Dent, v. 52, n. 1, jan./fev. 1998. Disponível em: http://www.apcd.org.br/biblioteca/revista/1998/jan_fev_98/orientando_paciente.asp. Acesso em: 22/12/03.

ORAL CANCER. Carcinogênese. Fatores de risco. Lesões precursoras. Lesões malignas. Auto-exame. Disponível em: http://www.oralcancer.cjb.net. Acesso em: 11/12/2002.

PARAJARA, F. Enfrentando o câncer bucal. Rev Assoc Paul Cir Dent, v. 53, n. 5, p. 353-360, set./out. 1999.

PIERRET, J. Les significations sociales de la santé: Paris, L'Essome, L'Heraut. In: AUGÉ, M.; HERLZLICH, C. Le sens du mal: anthropologie, historie, sologie de la maladie. Paris : Editions des Archives Contemporaines, 1986. p. 217-256.

PIERRET, J.; HERZLICH, C. Maladies d'hier, maladies d'aujourd'hui. Paris : Payot, 1984.

PIRES, A. B. et al. Diagnóstico do câncer de boca, responsabilidade do odontólogo. Trabalho apresentado no $5^{\mathrm{o}}$ OdontoRio, 2000. Disponível em: http://www.odontologia.com.br.

ROZEMBERG, B. Representação social de eventos somáticos ligados à esquistossomose. Cad Saúde Pública, v. 10, n. 1, p. 41, jan./mar. 1994.

SANTANA, J. P.; CHRISTÓFARO, M. A. C. Educação, trabalho e formação profissional em Saúde. Disponível em: www.pessoalsus.inf.br/. Acesso em: 11/12/2002.

SANTANA, J. P. et al. Formação profissional em Saúde: desafios para a universidade. Disponível em: http://www.abrasco.org.br. Acesso em: 11/12/2002.

SEIXAS, L. Alerta para o câncer bucal. Disponível em: http://www.odontologia.com.br/noticias.Asp?id=85. Acesso em: 14/02/01.

TORRES, I. A. Câncer de boca no Brasil - perfil atual do odontólogo frente ao problema. Saúde em Debate, n. 37, p. 44-47, dez. 1992.

UNFER, B.; SALIBA, O. Avaliação do conhecimento popular e práticas cotidianas em saúde bucal. Rev Saúde Pública, v. 34, n. 2, p. 190-195, 2000.

VERDÈS-LEROUX, J. Trabalhador social. Prática, ethos, formas de intervenção. São Paulo : Cortez Editora, 1986.

Aceito para publicação em 11/2003 